\title{
A spotlight on heightened T cell complexity and relevance in mucosal tissues
}

\author{
Jennifer M. Lund (iD) ${ }^{1,2}$ 压 \\ (c) The Author(s), under exclusive licence to Society for Mucosal Immunology 2022
}

Mucosal Immunology (2022) 15:377-378; https://doi.org/10.1038/s41385-022-00485-z

\section{INTRODUCTION}

Recent work has established the critical importance of tissueresident memory $\mathrm{T}$ cells $\left(\mathrm{T}_{\mathrm{RM}}\right)$ in protection from a variety of infections, malignancies, and other diseases. In this special review collection, we present five review articles that focus on mucosal tissue $T$ cells, both $T_{R M}$ and tissue regulatory $T$ cells.

The study of immunity within mucosal tissues has long been appreciated to be critical for leading to new clinical interventions for prevention and treatment of many diseases and infections of global health significance that impact these tissue sites. These include but are not limited to tumors in mucosal tissues, autoimmune conditions of the gastrointestinal (GI) or respiratory tracts, asthma, sexually transmitted infections, Gl infections, and respiratory infections such as influenza and coronaviruses. However, the technical difficulty in working with mucosal tissues from animal model systems, including relatively low immune cell yields from these non-lymphoid tissues, compounded by the complexity of obtaining human mucosal tissues in settings of both health and disease, has historically led to a relative paucity of studies of immune cells in mucosal barrier tissues as compared to immune cells from the circulation or lymphoid tissues. Despite these challenges, countless groups have focused on mucosal immunity, aided by recent advances in single-cell technologies such as high parameter flow cytometry and ssRNAseq approaches to secure unprecedented quantities of data from each rare cell recovered from these mucosal tissue locations. In particular, the past decade has seen an incredible advancement in the study of tissue-resident memory $T$ cells $\left(T_{R M}\right)$, or $T$ cells that reside in non-lymphoid tissues, including the mucosa, without recirculation ${ }^{1-7}$. In this review collection, we focus on these mucosal tissue $T$ cells to review the plethora of studies that have demonstrated the prominence of $T_{\mathrm{RM}}$ in aiding in protection from infection and disease.

\section{$T_{\text {RM }}$ IN THE RESPIRATORY TRACT NICHE}

The current COVID-19 pandemic has re-invigorated appreciation for the study of immunity in mucosal tissue compartments, with a particular spotlight on the respiratory tract. While vaccines have dramatically reduced severe disease and mortality associated with SARS-CoV-2 infection, the continual emergence of new variants of concern with differing transmissibility and ability to escape vaccine-induced and natural immunity underscores the possible advantages of mucosal vaccination that might better arm tissue lymphocytes, including $T_{R M}$, to combat infection at the portal of pathogen entry. In this issue's review by Zheng and Wakim, $T_{R M}$ within both the upper and lower respiratory tract are profiled ${ }^{8}$. The authors describe seminal studies that have elucidated the cues required for respiratory tract $\mathrm{T}_{\mathrm{RM}}$ development and maintenance, and they also highlight how respiratory tract $T_{R M}$ participate in immune surveillance against pathogen invasion and how these cells might be leveraged via vaccination. While respiratory tract $T_{R M}$ share many features with $T_{R M}$ in other barrier tissue sites, the focus on this unique microenvironmental niche may be critical when considering how to best prevent respiratory infections and associated disease.

\section{DIFFERENCES IN MOUSE AND HUMAN TISSUE T CELLS}

Study of immunity in small animal model systems has been critical to make rapid advances in our understanding, yet ultimately the motivation behind these discoveries is to improve human health outcomes, and so studies of human immunity are equally critical, albeit challenging. Thus, our collection includes a review by Lange and colleagues of human mucosal $T_{R M}$ in health and disease ${ }^{9}$. The authors discuss the current state of knowledge regarding human $T_{R M}$ function within the Gl tract, lung, head and neck, and female reproductive tract (FRT), as compared to what has been discovered using mouse model systems. $T_{R M}$ have been shown in each of these mucosal tissues to function in protection from pathogens as well as malignancies, and this focus on human $T_{R M}$ again highlights and explores how these cells can be best exploited therapeutically to limit human disease and suffering.

\section{MICROBIOTA-MEDIATED SHAPING OF TISSUE T CELLS}

One of many unique features of the mucosa that impacts mucosal immune cell development and homeostasis is the abundant microbiota present in these compartments compared to the circulation or lymphoid tissues. The presence of complex microbial populations of bacteria, fungi, viruses, and protists at mucosal sites leads to an abundance of microbiome-derived antigens and metabolites that in turn affect cells and processes of the immune system, including $T_{\mathrm{RM}}$. The importance of exposure of the host to a diverse array of microbes has been recently highlighted through studies of so-called "pet store mice"10,11. Compared to mice

\footnotetext{
Vaccine and Infectious Disease Division, Fred Hutchinson Cancer Research Center, Seattle, WA, USA. ${ }^{2}$ Department of Global Health, University of Washington, Seattle, WA, USA. 凶email: jlund@fredhutch.org
}

Received: 10 January 2022 Accepted: 13 January 2022

Published online: 23 February 2022 
continually housed in specific pathogen-free (SPF) facilities, feral or pet store mice have robust populations of effector and mucosal memory $\mathrm{T}_{\text {cells }}{ }^{10}$. This seminal study from Beura and colleagues not only provided new insight into how to improve mouse models to better mimic the human immune landscape, but also provided evidence for the hypothesis that the microbiota plays critical roles in shaping the $T_{R M}$ compartment. The review by Overacre-Delgoff and Hand includes discussion of studies in this emerging field that have demonstrated that the microbiota, including microbialderived metabolites and antigens, shapes CD8+and CD4+T development, survival, and function.

\section{A ROLE FOR TREGS IN TISSUE T CELL BIOLOGY}

It is well-established that $T_{R M}$ play critical roles in protection from infection and disease in mucosal barrier sites. This is at least in part due to their location at the portal of pathogen entry, where they are present and thus immediately poised to act, as well as their superior memory potential by which they can rapidly mediate their effector function. Thus, given that $\mathrm{T}_{\mathrm{RM}}$ are located at barrier sites of initial pathogen invasion and are armed and dangerous, it stands to reason that these powerful cells would be subject to regulation to minimize immune-mediated damage that could compromise critical mucosal tissue functions. In two reviews within our collection, we examine the role of regulatory $\mathrm{T}$ cells (Treg) in balancing the $\mathrm{T}_{\mathrm{RM}}$ response in mucosal tissues. Tregs are critically important in mediating peripheral tolerance, and can suppress various immune cells, including $T$ cells, through a variety of mechanisms. While Tregs were initially studied predominantly in lymphoid tissues, more recent work within the past 10 years has focused on the unique phenotypes and functions that nonlymphoid tissue Treg possess, including in mucosal tissues. Thus, Barros and colleagues discuss $\mathrm{T}_{\mathrm{RM}}$ and Treg populations within tissues, and explore the interplay between $T_{R M}$ and Treg ${ }^{12}$. In a separate review, Traxinger and colleagues summarize studies that have identified unique features and functions of mucosal tissue Tregs, including in the respiratory tract, Gl tract, and genitourinary tract in health and disease ${ }^{13}$. We hope and expect that future work will continue to advance our understanding of mucosal tissue $T$ cells and lead to new clinical strategies to prevent and treat infections and diseases by harnessing $\mathrm{T}_{\mathrm{RM}}$ and tissue Treg.

\section{REFERENCES}

1. Masopust, D., Jiang, J., Shen, H. \& Lefrancois, L. Direct analysis of the dynamics of the intestinal mucosa CD8 T cell response to systemic virus infection. J. Immunol. 166, 2348-2356 (2001).

2. Masopust, D., Vezys, V., Marzo, A. L. \& Lefrancois, L. Preferential localization of effector memory cells in nonlymphoid tissue. Science 291, 2413-2417 (2001).

3. Gebhardt, T. et al. Memory T cells in nonlymphoid tissue that provide enhanced local immunity during infection with herpes simplex virus. Nat. Immunol. 10, 524-530 (2009).

4. Jiang, $X$. et al. Skin infection generates non-migratory memory CD8+ T(RM) cells providing global skin immunity. Nature 483, 227-231 (2012).

5. Masopust, D. et al. Dynamic T cell migration program provides resident memory within intestinal epithelium. J. Exp. Med. 207, 553-564 (2010).

6. Teijaro, J. R. et al. Cutting edge: Tissue-retentive lung memory CD4 T cells mediate optimal protection to respiratory virus infection. J. Immunol. 187, 5510-5514 (2011).

7. Wakim, L. M., Woodward-Davis, A. \& Bevan, M. J. Memory T cells persisting within the brain after local infection show functional adaptations to their tissue of residence. Proc. Natl Acad. Sci. USA 107, 17872-17879 (2010).

8. Zheng, M. Z. M. \& Wakim, L. M. Tissue-resident memory T cells in the respiratory tract. Mucosal Immunol. (2021). Online ahead of print.

9. Lange, J., Rivera-Ballesteros, O. \& Buggert, M. Human mucosal tissue-resident memory T cells in health and disease. Mucosal Immunol. (2021). Online ahead of print.

10. Beura, L. K. et al. Normalizing the environment recapitulates adult human immune traits in laboratory mice. Nature 532, 512-516 (2016).

11. Hamilton, S. E. et al. New insights into the immune system using dirty mice. J. Immunol. 205, 3-11 (2020).

12. Barros, L., Ferreira, C. \& Veldhoen, M. The fellowship of regulatory and tissue-resident memory cells. Mucosal Immunol. 15, 64-73 (2022).

13. Traxinger, B. R., Richert-Spuhler, L. E. \& Lund J. M. Mucosal tissue regulatory T cells are integral in balancing immunity and tolerance at portals of antigen entry. Mucosal Immunol. (2021). Online ahead of print.

\section{COMPETING INTERESTS}

The author declares no competing interests.

\section{ADDITIONAL INFORMATION}

Correspondence and requests for materials should be addressed to Jennifer M. Lund.

Reprints and permission information is available at http://www.nature.com/reprints

Publisher's note Springer Nature remains neutral with regard to jurisdictional claims in published maps and institutional affiliations. 